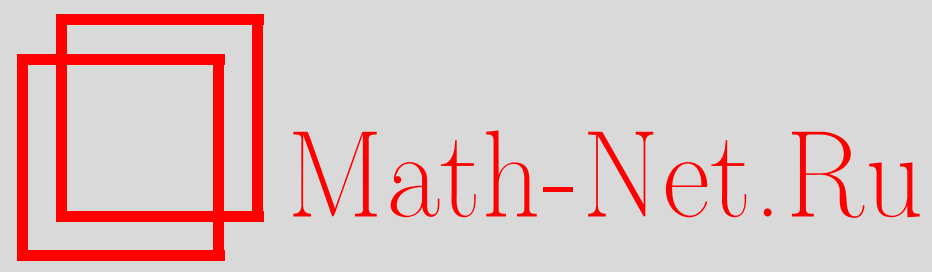

А. А. Туганбаев, Модули над кольцами эндоморфизмов, Матем. заметки, 2004, том 75, выпуск 6, 895-908

DOI: https://doi.org/10.4213/mzm79

Использование Общероссийского математического портала Math-Net.Ru подразумевает, что вы прочитали и согласны с пользовательским соглашением http://www.mathnet.ru/rus/agreement

Параметры загрузки:

IP: 54.196 .121 .252

26 апреля 2023 г., 12:44:07 
УДК 512.55

\title{
МОДУЛИ НАД КОЛЬЦАМИ ЭНДОМОРФИЗМОВ
}

\author{
А. А. Туганбаев
}

\begin{abstract}
Доказано, что $A$ - дистрибутивное справа кольцо тогда и только тогда, когда все квазиинъективные правые $A$-модули являются левыми модулями Безу над своими кольцами эндоморфизмов тогда и только тогда, когда каждое прямое слагаемое $N$ любого квазиинъективного правого $A$-модуля $M$, являющегося левьм $\operatorname{End}(M)$-модулем Безу, является левым $\operatorname{End}(N)$-модулем Безу. Если $A$-совершенное справа или слева кольцо, то все правые $A$-модули являются левыми модулями Безу над своими кольцами эндоморфизмов тогда и только тогда, когда все правые $A$-модули являются дистрибутивными левыми модулями над своими кольцами эндоморфизмов тогда и только тогда, когда $A$ - дистрибутивное кольцо.
\end{abstract}

Библиографоия: 6 названий.

Все кольца предполагаются ассоциативными и с ненулевой единицей. Модуль, у которого все конечно порожденные подмодули являются циклическими, назьвается модулем Безу. Модуль $M$ назьвается дистрибутивным, если решетка всех его подмодулей $\operatorname{Lat}(M)$ дистрибутивна. Слова типа "дистрибутивное кольцо" означают, что соответствующие условия выполнены справа и слева. В теореме 8.12 из [1] доказано, что правая дистрибутивность кольца $A$ равносильна тому, что инъективная оболочка любого простого правого $A$-модуля является левьм модулем Безу над своим кольцом эндоморфизмов.

Основными результатами данной работы являются теоремы 1 и 2 .

Теорема 1. Для кольиа А равносильны следуюшие условия:

(1) $A$ - дистрибутивное справа кольцо;

(2) каждый инбективный правый $A$-модуль $M$ является левым $\operatorname{End}(M)$-модулем Безу;

(3) кажсдый квазиинбективный правый $A$-модуль $M$ является левым $\operatorname{End}(M)$ модулем Безу;

(4) каждое прямое слагаемое $N$ любого квазиинвективного правого $A$-модуля $M$, являюшегося левим $\operatorname{End}(M)$-модулем Безу, является левым $\operatorname{End}(N)$ модулем Безу.

Работа поддержана Российским фондом фундаментальных исследований, проект № 99-01-00382. 
ТЕорема 2. Для совершенного справа или слева кольца А равносильны следующие условия:

(1) все правые А-модули являются левыми модулями Безу над своими кольиами әндоморфизмов;

(2) все правые $A$-модули являются дистрибутивными левыми модулями над своими кольцами эндоморфизмов;

(3) $A$ - дистрибутивное кольио.

В связи с теоремой 1 в примере 1.9 показано, что существует инъективный правый модуль $M$, которьй является левым $\operatorname{End}(M)$-модулем Безу, причем $M$ имеет свободное циклическое инъективное прямое слагаемое $N$, которое не является левым $\operatorname{End}(N)$-модулем Безу.

В связи с теоремой 2 заметим, что кольцо целых чисел $\mathbb{Z}$ является дистрибутивным кольцом, причем не все $\mathbb{Z}$-модули являются дистрибутивными модулями над своими кольцами эндоморфизмов (см. пример 2.13).

Доказательства теорем 1 и 2 разбиты на ряд утверждений. Кольцо $A$ называется полусовершенным, если фактор-кольцо кольца $A$ по его радикалу Джекобсона $J(A)$ является артиновым кольцом. Кольцо $A$ называется совершенным справа, если $A-$ полусовершенное кольцо и для любой последовательности $\left\{a_{i}\right\}_{i=1}^{\infty}$ элементов из $J(A)$ существует такое натуральное число $n$, что $a_{n} a_{n-1} \ldots a_{2} a_{1}=0$. Модуль $M$ назьвается иепны.м, если любые два его подмодуля сравнимы по включению (т.е. решетка Lat $(M)$ является цепью). Модуль $M$ назьвается полуцепнылм, если $M$ - прямая сумма цепных модулей. Модуль назьвается равномерным, если любые два его ненулевых подмодуля имеют ненулевое пересечение. Пусть $M$ - модуль. Модуль $N$ называется инғективным относительно $M$ (или $M$-инвективным), если для любого подмодуля $M^{\prime}$ модуля $M$ каждьй гомоморфизм $M^{\prime} \rightarrow N$ продолжается до гомоморфизма $M \rightarrow N$. Модуль называется квазиинғективным, если он инъективен относительно себя. Правый модуль $N$ над кольцом $A$ назьвается инбективным, если $N$ инъективен относительно всех правых $A$-модулей. Если $N$ - инъективньй модуль, являющийся существенным расширением своего подмодуля $N^{\prime}$, то $N$ называется инбективной оболочкой модуля $N^{\prime}$. Инъективная оболочка определена однозначно с точностью до изоморфизма. Подмодуль фактор-модуля модуля $M$ назьвается подфактором модуля $M$.

\section{1. Доказательство теоремы 1.}

Лемма 1.1. Пусть $X$ и $M$ - правые модули над кольцом $A$ и левый $\operatorname{End}(M)-м о-$ дуль $\operatorname{Hom}(X, M)$ является модулем Безу. Тогда

(1) для любых двух гомоморфизмов $f$ и $g$ из $X$ в $M$ существует такой гомоморфизм $h: X \rightarrow M$, что $\operatorname{Ker}(f) \cap \operatorname{Ker}(g)=\operatorname{Ker}(h)$;

(2) если модуль $M$ равномерен, то для любых двух гомоморфизмов $f$ и $g$ из $X$ в $M$ модули $\operatorname{Ker}(f)$ и $\operatorname{Ker}(g)$ сравнимы по включению;

(3) для любого вполне инвариантного подмодуля $N$ модуля $M$ аддитивная группа $\operatorname{Hom}(X, N)$ является левым $\operatorname{End}(M)$-модулем Безу;

(4) если $M-$ ингективный модуль и $N$ - квазиинбективный существенный подмодуль модуля $M$, то левый $\operatorname{End}(N)$-модуль $\operatorname{Hom}(X, N)$ является модулем Безу. 
ДОКАЗАТЕЛЬСТво. Пусть $R \equiv \operatorname{End}(M)$.

(1) По условию $R f+R g$ - циклический левьй $R$-модуль. Поэтому существуют такие эндоморфизмы $s, t, u$ и $v$ модуля $M$, что

$$
f=s(u f+v g) \quad \text { и } \quad g=t(u f+v g) .
$$

Тогда $\operatorname{Ker}(f) \cap \operatorname{Ker}(g)=\operatorname{Ker}(u f+v g)$ и можно положить $h \equiv u f+v g$.

(2) Пусть

$$
\pi: X \rightarrow X /(\operatorname{Ker}(f) \cap \operatorname{Ker}(g))
$$

- естественный эпиморфизм. По (1) существует такой гомоморфизм $h: X \rightarrow M$, что

$$
\operatorname{Ker}(h)=\operatorname{Ker}(f) \cap \operatorname{Ker}(g) .
$$

Гомоморфизм $h$ индуцирует мономорфизм $\bar{h}: \pi(X) \rightarrow M$.

Допустим, что модули $\operatorname{Ker}(f)$ и $\operatorname{Ker}(g)$ не сравнимы по включению. Тогда $\pi(\operatorname{Ker}(f))$ и $\pi(\operatorname{Ker}(g))$ - ненулевые подмодули модуля $\pi(X)$ и

$$
\pi(\operatorname{Ker}(f)) \cap \pi(\operatorname{Ker}(g))=0 .
$$

Так как $\bar{h}$ - мономорфизм, то $\bar{h} \pi(\operatorname{Ker}(f))$ и $\bar{h} \pi(\operatorname{Ker}(g))$ - ненулевые подмодули равномерного модуля $M$ и

$$
h \pi(\operatorname{Ker}(f)) \cap h \pi(\operatorname{Ker}(g))=0 .
$$

Получено противоречие.

(3) Так как $N$-вполне инвариантньй подмодуль модуля $M$, то левьй $\operatorname{End}(N)$-модуль $\operatorname{Hom}(X, N)$ является левым $\operatorname{End}(M)$-модулем. Поэтому $\operatorname{Hom}(X, N)$ - подмодуль левого $\operatorname{End}(M)$-модуля Безу. Следовательно, $\operatorname{Hom}(X, N)$ - левый $\operatorname{End}(M)$-модуль Безу.

(4) Так как $M$ - инъективньй модуль и $N$ - квазиинъективньй существенньй подмодуль модуля $M$, то $M$ - инъективная оболочка квазиинъективного модуля $N$. Поэтому $N$ - вполне инвариантный подмодуль модуля $M$ (см. предложение 19.2 из [2]). По (3) $\operatorname{Hom}(X, N)$ - левьй $\operatorname{End}(M)$-модуль Безу. Кроме того, каждый эндоморфизм модуля $N$ продолжается до эндоморфизма инъективного модуля $M$. Следовательно, $\operatorname{Hom}(X, N)$ - левьй $\operatorname{End}(N)$-модуль Безу.

Лемма 1.2. Пусть $A-$ кольцо, $X-$ правый $A$-модуль, $S$ - простой правый $A$-модуль и $M$ - инбективная оболочка модуля $S$. Тогда равносильны следующие условия:

(1) $\operatorname{Hom}(X, M)$ - иепной левый $\operatorname{End}(M)$-модуль;

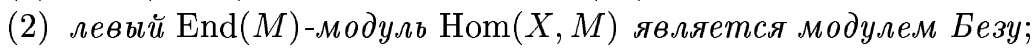

(3) для любых двух гомоморфизмов $f$ u $g$ из $X$ в $M$ модули $\operatorname{Ker}(f) u \operatorname{Ker}(g)$ сравнимы по включению;

(4) $X$ не имеет таких подфакторов $S_{1} \oplus S_{2}$, что $S_{1} \cong S_{2} \cong S$. 
ДокАЗАТЕЛьСТво. Импликация $(1) \Longrightarrow(2)$ очевидна.

$(2) \Longrightarrow(3)$ Так как $M$ - инъективная оболочка простого модуля $S$, то $M$ - равномерный модуль. Поэтому утверждение следует из леммы 1.1(2).

$(3) \Longrightarrow(4)$ Допустим противное. Тогда модуль $X$ имеет такие подмодули $Y$ и $Z$, что $Y /(Y \cap Z) \cong Z /(Y \cap Z) \cong S$. Поэтому сушествуют такие эпиморфизмы $f_{1}, g_{1}: Y+Z \rightarrow S$, что $\operatorname{Ker}\left(f_{1}\right)=Z$ и $\operatorname{Ker}\left(g_{1}\right)=Y$. Так как $S$ - подмодуль инъективного модуля $M$, то гомоморфизмы $f_{1}$ и $g_{1}$ продолжаются до гомоморфизмов $f, g: X \rightarrow M$. По условию модули $\operatorname{Ker}(f)$ и $\operatorname{Ker}(g)$ сравнимы по включению. Например, пусть $\operatorname{Ker}(f) \subseteq \operatorname{Ker}(g)$. Тогда $Z=\operatorname{Ker}\left(f_{1}\right) \subseteq \operatorname{Ker}(f) \subseteq \operatorname{Ker}(g)$. Поэтому $S=g_{1}(Y+Z)=g_{1}(Z)=g(Z)=0$. Получено противоречие.

$(4) \Longrightarrow(3)$ Допустим противное. Тогда существуют такие гомоморфизмы $f, g$ : $X \rightarrow S$ и подмодули $\bar{Y}$ и $\bar{Z}$ модуля $X$, что

$$
f(\bar{Z})=g(\bar{Y})=0, \quad f(\bar{Y}) \neq 0, \quad g(\bar{Z}) \neq 0 .
$$

Так как $M$ - существенное расширение простого модуля $S$, то $S \subseteq f(\bar{Y}) \cap g(\bar{Z})$. Поэтому существуют такие подмодули $Y \subseteq \bar{Y}$ и $Z \subseteq \bar{Z}$, что $S=f(Y)=g(Z)$. Кроме того, $f(Y \cap Z)=g(Y \cap Z)=0$. Поэтому существуют эпиморфизмы $Y /(Y \cap Z) \rightarrow S$ и $Z /(Y \cap Z) \rightarrow S$. Следовательно, модуль $X$ имеет такой подфактор $S_{1} \oplus S_{2}$, что $S_{1} \cong S_{2} \cong S$.

$(3) \Longrightarrow(1)$ Пусть $R \equiv \operatorname{End}(M)$ и $f, g \in \operatorname{Hom}(X, M)$. По условию либо $\operatorname{Ker}(f) \subseteq$ $\operatorname{Ker}(g)$, либо $\operatorname{Ker}(g) \subseteq \operatorname{Ker}(f)$. Допустим, что $\operatorname{Ker}(f) \subseteq \operatorname{Ker}(g)$. Тогда существует такой гомоморфизм $\bar{h}: f(X) \rightarrow g(X)$, что $\bar{h} f(x)=g(x)$. Так как $M$ - инъективный модуль, то гомоморфизм $\bar{h}$ продолжается до эндоморфизма $h$ модуля $M$. Поэтому $g=h f \in R f$. Аналогично, если $\operatorname{Ker}(g) \subseteq \operatorname{Ker}(f)$, то $f \in R g$. Поэтому $\operatorname{Hom}(X, M)$ - цепной левый $\operatorname{End}(M)$-модуль.

Лемма 1.3. Для правого модуля $X$ над кольиом А равносильны следующие условия:

(1) $X-$ - дстрибутивный модуль;

(2) модуль $X$ не имеет таких подфакторов $S_{1} \oplus S_{2}$, ито $S_{1}$ и $S_{2}-$ изоморфные простые модули;

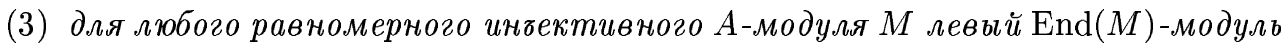
$\operatorname{Hom}(X, M)$ является модулем Безу;

(4) для любого равномерного инбективного $A$-модуля $M$ левый $\operatorname{End}(M)$-модуль $\operatorname{Hom}(X, M)$ является цепным модулем.

ДокАЗАТЕЛЬСтво. Эквивалентность условий (1) и (2) доказана в [3].

Эквивалентность условий (2), (3) и (4) следует из леммы 1.2.

ЛЕмма 1.4 [4, п. 16.2]. Пусть $U-$ модуль и $M-U$-инбективный модуль. Тогда $M$ ингективен относительно любого подфактора модуля $U$.

Кроме того, если $V$ - модуль и $M$ инвективен относительно $V$, то $M$ $(U \oplus V)$-ингективный модуль. 


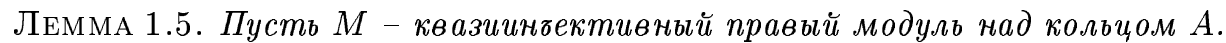

(1) Если $X-$ правый $A$-модуль, то левый $\operatorname{End}(M)$-модуль $\operatorname{Hom}(X, M)$ является модулем Безу тогда и только тогда, когда для любых двух гомоморфизмов $f$ и $g$ из $X$ в $M$ существует такой гомоморфизм $h: X \rightarrow M$, что $\operatorname{Ker}(f) \cap \operatorname{Ker}(g)=\operatorname{Ker}(h)$.

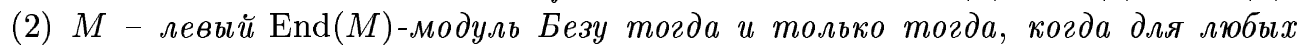
двух әлементов $u, v \in M$ существует такой әлемент $w \in M$, что

$$
r(u) \cap r(v)=r(w) .
$$

(3) Если модуль $M$ содержит изоморфные копии всех ииклических правых $A$-мо-

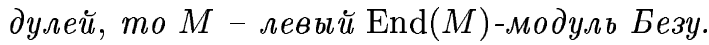

ДокАЗАТЕЛЬСтво. (1) Достаточность следует из леммы 1.1(1).

Необходимость. Пусть $f, g \in \operatorname{Hom}(X, M)$. По условию существует такой гомоморфизм $h: X \rightarrow M$, что

$$
\operatorname{Ker}(f) \cap \operatorname{Ker}(g)=\operatorname{Ker}(h) .
$$

Так как $M-M$-инъективный модуль, то по лемме 1.4 модуль $M$ инъективен относительно своих подмодулей $f(X)$ и $g(X)$. По лемме 1.4 модуль $M$ инъективен относительно внешней прямой суммы $f(X) \oplus g(X)$. Так как $\operatorname{Ker}(f) \cap \operatorname{Ker}(g)=\operatorname{Ker}(h)$, то существует такой мономорфизм

$$
s: h(X) \rightarrow f(X) \oplus g(X)
$$

что

$$
s(h(x))=(f(x), g(x)) \quad \text { для всех } x \in X .
$$

Так как $M$ инъективен относительно $f(X) \oplus g(X)$, то изоморфизм $s^{-1}: s(h(X)) \rightarrow h(X)$ продолжается до гомоморфизма

$$
t: f(X) \oplus g(X) \rightarrow M
$$

Так как $M$ инъективен относительно $f(X)$ и $g(X)$, то гомоморфизмы $\left.t\right|_{f(X)}: f(X) \rightarrow M$ и $\left.t\right|_{g(X)}: g(X) \rightarrow M$ продолжаются до эндоморфизмов $u$ и $v$ модуля $M$. Пусть $R \equiv$ $\operatorname{End}(M)$. Если $x \in X$, то

$$
\begin{aligned}
(u f+v g)(x) & =u f(x)+v g(x)=t f(x)+\operatorname{tg}(x) \\
& =t((f(x), g(x)))=s^{-1}((f(x), g(x)))=h(x) .
\end{aligned}
$$

Поэтому $h=u f+v g \in R f+R g$.

Так как $\operatorname{Ker}(h) \subseteq \operatorname{Ker}(f)$ и $\operatorname{Ker}(h) \subseteq \operatorname{Ker}(g)$, то существуют такие эпиморфизмы $w_{1}: h(X) \rightarrow f(X)$ и $w_{2}: h(X) \rightarrow g(X)$, что

$$
w_{1}(h(x))=f(x) \quad \text { и } \quad w_{2}(h(x))=g(x) \quad \text { для всех } \quad x \in X .
$$

Так как $M$ - квазиинъективньй модуль, то гомоморфизмы $w_{1}$ и $w_{2}$ продолжаются до эндоморфизмов $z_{1}$ и $z_{2}$ модуля $M$. Тогда $f=z_{1} h$ и $g=z_{2} h$. Поэтому

$$
R f+R g \subseteq R h \subseteq R f+R g
$$


Так как $R f+R g=R h$, то $\operatorname{Hom}(X, M)$ - левьй $R$-модуль Безу.

(2) По (1) $\operatorname{Hom}\left(A_{A}, M\right)$ - левый $\operatorname{End}(M)$-модуль Безу тогда и только тогда, когда для любых двух элементов $u, v \in M$ существует такой элемент $w \in M$, что $r(u) \cap r(v)=r(w)$. Кроме того, существует естественный изоморфизм $M \cong \operatorname{Hom}\left(A_{A}, M\right)$.

(3) По (2) достаточно показать, что для любых двух элементов $u, v \in M$ существует такой элемент $w \in M$, что

$$
r(u) \cap r(v)=r(w) .
$$

Это следует из того, что по условию модуль $M$ содержит изоморфную копию циклического модуля $A_{A} /(r(u) \cap r(v))$.

ЛЕмма 1.6. Пусть $X-$-истрибутивный правый модуль, $\bar{X}-$ подфактор модуля $X, M-X$-ингективный правый модуль и существуют такие гомоморфизмы $f, g \in \operatorname{Hom}(\bar{X}, M)$, ито $\operatorname{Ker}(f) \cap \operatorname{Ker}(g)=0$.

Тогда существует мономорфизм $h: \bar{X} \rightarrow M$.

ДокАЗАтЕльство. Модуль $\bar{X}$ содержит подмодуль $\operatorname{Ker}(f) \oplus \operatorname{Ker}(g)$. Существует такой подмодуль $P$ модуля $\bar{X}$, что $P \cap(\operatorname{Ker}(f) \oplus \operatorname{Ker}(g))=0$ и $P \oplus \operatorname{Ker}(f) \oplus \operatorname{Ker}(g)-$ существенный подмодуль в $\bar{X}$. Существует такой гомоморфизм

$$
h^{\prime}: P \oplus \operatorname{Ker}(f) \oplus \operatorname{Ker}(g) \rightarrow M,
$$

что $h^{\prime}$ совпадает с $g$ на модуле $P \oplus \operatorname{Ker}(f)$ и $h^{\prime}$ совпадает с $f$ на модуле $\operatorname{Ker}(g)$. По лемме $1.4 M-\bar{X}$-инъективньй модуль. Поэтому $h^{\prime}$ продолжается до гомоморфизма $h: \bar{X} \rightarrow M$ с ядром $X$.

Так как

$$
g(\operatorname{Ker}(h) \cap(P \oplus \operatorname{Ker}(f)))=h(\operatorname{Ker}(h) \cap(P \oplus \operatorname{Ker}(f)))=0,
$$

TO

$$
\operatorname{Ker}(h) \cap(P \oplus \operatorname{Ker}(f)) \subseteq \operatorname{Ker}(g) \cap(P \oplus \operatorname{Ker}(f))=0 .
$$

Кроме того,

$$
\operatorname{Ker}(h) \cap \operatorname{Ker}(g) \subseteq \operatorname{Ker}(f) \cap \operatorname{Ker}(g)=0 .
$$

Так как $\bar{X}$ - дистрибутивньй модуль, то

$$
\operatorname{Ker}(h) \cap(P \oplus \operatorname{Ker}(f) \oplus \operatorname{Ker}(g))=\operatorname{Ker}(h) \cap(P \oplus \operatorname{Ker}(f))+\operatorname{Ker}(h) \cap \operatorname{Ker}(g)=0
$$

и $h$ - требуемый мономорфизм.

ПРЕДЛОЖЕНИЕ 1.7. Пусть $X-$ правый модуль над кольцом А. Тогда равносильны следующие условия:

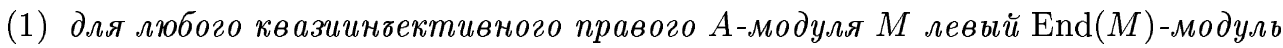
$\operatorname{Hom}(X, M)$ является модулем Безу;

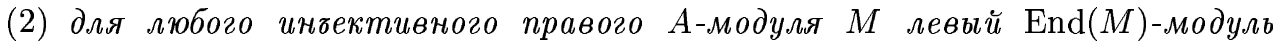
$\operatorname{Hom}(X, M)$ является модулем Безу;

(3) $X-$ - истрибутивный модуль. 
ДокАЗАТЕЛЬСТво. Импликация (1) $\Longrightarrow(2)$ очевидна.

Импликация $(2) \Longrightarrow(1)$ следует из леммы $1.1(4)$ и того, что каждый модуль обладает инъективной оболочкой.

Импликация $(2) \Longrightarrow(3)$ следует из леммы 1.3 .

$(3) \Longrightarrow(2)$ Пусть $f, g \in \operatorname{Hom}(X, M)$. По лемме $1.5(1)$ достаточно доказать, что существует такой гомоморфизм $h: X \rightarrow M$, что

$$
\operatorname{Ker}(f) \cap \operatorname{Ker}(g)=\operatorname{Ker}(h) .
$$

Обозначим через $\bar{X}$ дистрибутивньй модуль $X /(\operatorname{Ker}(f) \cap \operatorname{Ker}(g))$. Гомоморфизмы $f$ и $g$ индуцируют такие гомоморфизмы $\bar{f}, \bar{g} \in \operatorname{Hom}(\bar{X}, M)$, что $\operatorname{Ker}(\bar{f}) \cap \operatorname{Ker}(\bar{g})=0$. По лемме 1.6 существует мономорфизм $\bar{h}: \bar{X} \rightarrow M$. Если $\pi: X \rightarrow \bar{X}$ - естественный эпиморфизм и $h \equiv \bar{h} \pi$, то $h$ - требуемьй гомоморфизм.

ПРЕДЛОЖЕНИЕ 1.8. Пусть $A-$ кольио, $\left\{B_{i}\right\}_{i \in I}-$ множсество всех правых идеалов кольиа $A$ и $M$ - инбективная оболочка модуля $\bigoplus_{i \in I} A_{A} / B_{i}$. Тогда равносильны следующие условия:

(1) $A$ - дистрибутивное справа кольио;

(2) все инбективные правые А-модули являются левыми модулями Безу над своими кольиами әндоморфизмов;

(3) все квазиинбективные правые А-модули являются левыми модулями Безу над своими кольцами әндоморфизмов;

(4) каждое прямое слагаемое $N$ любого квазиинбективного правого $A$-модуля Безу $M$ является левым $\operatorname{End}(N)$-модулем Безу;

(5) все прямые слагаемые инбективного модуля $M$ являются левыми модулями Безу над своими кольцами әндоморфизмов;

(6) для каждого простого правого $A$-модуля $S$ индективная оболочка модуля $S$ является левым модулем Безу над своим кольчом әндоморфизмов.

ДокАЗАТЕльСтво. Для каждого правого $A$-модуля $N$ существует естественньй изоморфизм $N \cong \operatorname{Hom}\left(A_{A}, N\right)$. Поэтому эквивалентность условий $(1),(2),(3)$ и (6) следует из предложения 1.7 .

Импликация $(3) \Longrightarrow(4)$ следует из того, что любое прямое слагаемое квазиинъективного модуля является квазиинъективным модулем.

Импликация $(4) \Longrightarrow(5)$ следует из того, что по лемме $1.5(3)$ инъективный модуль $M$ является левьм $\operatorname{End}(M)$-модулем Безу.

Импликация $(5) \Longrightarrow(6)$ следует из того, что все инъективные оболочки простых правых $A$-модулей изоморфны прямым слагаемым инъективного модуля $M$.

ОКОНЧАНИЕ ДОКАЗАТЕЛЬСТВА ТЕОРЕМЫ 1 . Теорема 1 следует из предложения 1.8.

ПримеР 1.9. Сушествует инъективньй правый модуль $M$, который является левым $\operatorname{End}(M)$-модулем Безу, причем $M$ имеет свободное циклическое инъективное прямое слагаемое $N$, которое не является левым $\operatorname{End}(N)$-модулем Безу. 
ДоКАЗАТЕЛЬСТво. Пусть $F$ - поле из двух элементов, $\langle x\rangle$ и $\langle y\rangle-$ две циклические групшы порядка 2 с образуюшими $x$ и $y, G$ - прямое произведение групп $\langle x\rangle$ и $\langle y\rangle$, $A=F[G]-$ групповое кольцо. Тогда $A$ - коммутативное конечное самоинъективное кольцо и его 2 -порожденный идеал $(1-x) A+(1-y) A$ не является главным. Поэтому $N \equiv A_{A}-$ свободньй циклический инъективный правый модуль, который не является левым $\operatorname{End}(N)$-модулем Безу. Пусть $\left\{B_{i}\right\}_{i \in I}$ - множество всех правых идеалов кольца $A$ и $M$ - инъективная оболочка модуля $\oplus_{i \in I} A_{A} / B_{i}$. По лемме $1.5(3) M$ - левый $\operatorname{End}(M)$-модуль Безу. Кроме того, инъективньй циклический модуль $N$ изоморфен прямому слагаемому модуля $M$.

ЗАмЕчАниЕ 1.10. (1) Если $N$ - квазиинъективньй правый модуль, то $\operatorname{End}(N)$ - левое кольцо Безу тогда и только тогда, когда для любых двух эндоморфизмов $f$ и $g$ модуля $N$ сушествует такой эндоморфизм $h$ модуля $N$, что $\operatorname{Ker}(f) \cap \operatorname{Ker}(g)=\operatorname{Ker}(h)$.

(2) Если $N$ - дистрибутивньй квазиинъективный правьй модуль, то $\operatorname{End}(N)$ - левое кольцо Безу.

ДоКАЗАТЕЛЬСТво. (1) следует из леммы 1.5(1) при $M=N$.

(2) следует из предложения 1.7 при $M=N$.

\section{2. Доказательство теоремы 2.}

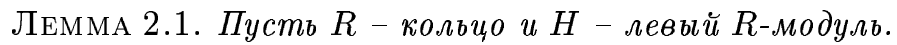

(1) $H$ - дистрибутивный $R$-модуль тогда и только тогда, когда для любых әлементов $f, g \in H$ существует такой әлемент $s \in R$, что $s f,(1-s) g \in R f \cap R g$.

(2) Eсли $S$ - унитарное подкольцо кольца $R$ и естественный $S$-модуль $H$ дистрибутивен, то $H$ - дистрибутивный $R$-модуль.

(3) Если $H$ - дистрибутивный $R$-модуль и $е$-ненулевой идемпотент кольца $R$, то ен - дистрибутивный еRе-модуль.

ДоказАтельство. (1) Докажем достаточность. Так как

$$
f+g \in R(f+g) \cap R(f+g)=R(f+g) \cap R f+R(f+g) \cap R g,
$$

то существуют такие элементы $s, t \in R$, что $f+g=t(f+g)+s(f+g), t(f+g) \in R f$ и $s(f+g) \in R g$. Тогда $t g=t(f+g)-t f \in R f \cap R g$ и $s f=s(f+g)-s g \in R f \cap R g$. Так как $(1-s-t)(f+g)=0$, то

$$
(1-s) g=(1-s-t)(f+g)-(1-s-t) f+t g=(s+t-1) f+t g \in R f \cap R g .
$$

Необходимость. Пусть $F, G$ и $Z$ - подмодули модуля $H, f \in F, g \in G, z \in Z$ и $z=f+g \in(F+G) \cap Z$. Достаточно доказать включение

$$
z \in R f \cap R z+R g \cap R z .
$$

По условию сушествует такой элемент $s \in R$, что $s f,(1-s) g \in R f \cap R g$. Тогда

$$
(1-s) z=(1-s) f+(1-s) g \in R f \cap R z, \quad s z=s f+s g \in R g \cap R z
$$

и $z=(1-s) z+s z \in R f \cap R z+R g \cap R z$.

(2) Утверждение следует из (1).

(3) Пусть $e f, e g \in e H$. По (1) сушествует такой элемент $s \in R$, что $s e f,(1-s) e g \in$ $R e f \cap R e g$. Тогда $e s e \cdot f,(e-e s e) \cdot e g \in e R e \cdot e f \cap e R e \cdot e g$. Кроме того, $e$ - единица кольца $e R e$. Применяя (1) к $e R e$-модулю $e H$, получаем, что $e H$ - дистрибутивньй $e R e$-модуль. 
Лемма 2.2. Пусть $R$ - прямое произведение колеи $R_{i}, i \in I$, и пусть для каждого $i \in I$ дан левый $R_{i}$-модуль $H_{i}$. Кроме того, пусть $H$ и $K$-соответственно прямое произведение и прямая сумма всех аддитивных групп $H_{i}$, pассматриваемые как естественный левый $R$-модуль.

(1) Если все левые $R_{i}$-модули $H_{i}$ являются модулями Безу, то $H-R$-модуль Безу.

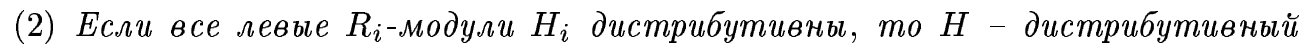
$R-\mathcal{м о д у л ь . ~}$

(3) Если все левье $R_{i}$-модули $H_{i}$ являются модулями Безу, то $K-R$-модуль Безу.

(4) Если все левые $R_{i}$-модули $H_{i}$ дистрибутивны, то $K$ - дистрибутивный $R-\mathcal{м о д у л ь . ~}$

ДоказАтЕЛьство. (1) Пусть $f, g \in H, f=\left(f_{i}\right)_{i \in I}, g=\left(g_{i}\right)_{i \in I}$, где $f_{i}, g_{i} \in H_{i}$. Так как все $R_{i}$-модули $H_{i}$ являются модулями Безу, то для каждого $i \in I$ существуют такие элементы $s_{i}, t_{i}, u_{i}, v_{i} \in R_{i}$, что $f_{i}=s_{i}\left(u_{i} f_{i}+v_{i} g_{i}\right)$ и $g_{i}=t_{i}\left(u_{i} f_{i}+v_{i} g_{i}\right)$. Пусть $s \equiv\left(s_{i}\right)_{i \in I} \in R, t \equiv\left(t_{i}\right)_{i \in I} \in R, u \equiv\left(u_{i}\right)_{i \in I} \in R$ и $v \equiv\left(v_{i}\right)_{i \in I} \in R$. Тогда $f=s(u f+v g)$ и $g=t(u f+v g)$. Поэтому $R f+R g=R(u f+v g)$ и $H-R$-модуль Безу.

(2) Пусть $f, g \in H, f=\left(f_{i}\right)_{i \in I}, g=\left(g_{i}\right)_{i \in I}$, где $f_{i}, g_{i} \in H_{i}$. Для каждого $i \in I$ обозначим через $1_{i}$ единицу кольца $R_{i}$. Так как все $R_{i}$-модули $H_{i}$ дистрибутивны, то по лемме 2.1(1) для каждого $i \in I$ сушествуют такие элементы $s_{i}, t_{i}, u_{i}, v_{i} \in R_{i}$, что $s_{i} f_{i}=t_{i} g_{i}$ и $\left(1-s_{i}\right) g_{i}=u_{i} f_{i}$. Пусть $s \equiv\left(s_{i}\right)_{i \in I} \in R, t \equiv\left(t_{i}\right)_{i \in I} \in R$ и $u \equiv\left(u_{i}\right)_{i \in I} \in R$. Тогда $s f=t g \in R f \cap R g$ и $(1-s) g=u f \in R f \cap R g$. По лемме 2.1(1) $H$ - дистрибутивный $R$-модуль.

(3) и (4) Доказательство аналогично доказательству утверждений (1) и (2).

Лемма 2.3. Пусть $A-к о л ь и о, ~\left\{H_{i}\right\}_{i \in I}$ - некоторое множество правых $A$-модулей, $H \equiv \prod_{i \in I} H_{i} u K \equiv \prod_{i \in I} H_{i}$.

(1) Если все $H_{i}$ являются левыми $\operatorname{End}\left(H_{i}\right)$-модулями Безу, то $H$ и $K$-левые

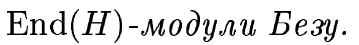

(2) Если все левые $\operatorname{End}\left(H_{i}\right)$-модули $H_{i}$ дистрибутивны, то $H$ и $K$ - дистрибу-

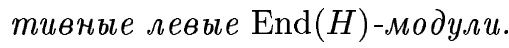

ДокАЗАтЕльство. Пусть $S$ - прямое произведение всех колец $\operatorname{End}\left(H_{i}\right)$. Кольцо $S$ может рассматриваться естественным образом как унитарное подкольцо кольца $\operatorname{End}(H)$.

(1) По лемме 2.2(1) и лемме $2.2(3) H$ и $K$ - левые модули Безу над подкольцом $S$ кольца $\operatorname{End}(H)$. Поэтому $H$ и $K$ - левые $\operatorname{End}(H)$-модули Безу.

(2) По лемме 2.2(2) и лемме 2.2(4) $H$ и $K$ - дистрибутивные левые модули над унитарным подкольцом $S$ кольца $\operatorname{End}(H)$. По лемме 2.1(2) $H$ и $K$ - дистрибутивные левые $\operatorname{End}(H)$-модули.

ЛЕмма 2.4. Пусть $X$ и $M$ - такие правые модули над кольиом $A$, что левый $\operatorname{End}(M)$-модуль $\operatorname{Hom}(X, M)$ дистрибутивен.

(1) Если $N$ - ненулевое прямое слагаемое модуля $M$, то левый $\operatorname{End}(N)$-модуль $\operatorname{Hom}(X, N)$ дистрибутивен.

(2) Если $X=Y \oplus Z$ и существуют такие гомоморфизмы $f, g: X \rightarrow M$, что $\operatorname{Ker}(f)=Z u \operatorname{Ker}(g)=Y$, то гомоморфизм $f-g: X \rightarrow M$ является мономорфизмом. 
(3) Если модуль $M$ равномерен $и X=Y \oplus Z$, где $Y \cong Z \neq 0$, то любой ненулевой гомоморфизм $Y \rightarrow M$ имеет ненулевое ядро.

(4) Для любого вполне инвариантного подмодуля $N$ модуля $M$ аддитивная группа $\operatorname{Hom}(X, N)$ является дистрибутивным левым $\operatorname{End}(M)$-модулем.

(5) Если $M-$ инвективный модуль и $N$ - квазиинвективный существенный подмодуль модуля $M$, то левый $\operatorname{End}(N)$-модуль $\operatorname{Hom}(X, N)$ дистрибутивен.

ДокАЗАТЕЛЬСТво. Положим $R \equiv \operatorname{End}(M)$.

(1) Существует такой идемпотент $е$ кольца $R$, что $N=e M$. Тогда $e R e=\operatorname{End}(N)$ и

$$
\operatorname{Hom}(X, N)=e \cdot \operatorname{Hom}(X, M)
$$

Поэтому утверждение следует из леммы 2.1(3).

(2) По лемме 2.1(1) существуют такие эндоморфизмы $s, t$ и $v$ модуля $M$, что $s f=t g$ и $(1-s) g=v f$. Тогда $X=\operatorname{Ker}(f)+\operatorname{Ker}(g) \subseteq \operatorname{Ker}(s f)$ и $X \subseteq \operatorname{Ker}((1-s) g)$. Поэтому $s f \equiv 0$ и $(1-s) g \equiv 0$. Поэтому $f \equiv(1-s) f$ и $g \equiv s g$. Пусть $x \in \operatorname{Ker}(f-g)$. Тогда $f(x)=g(x)$ и $g(x)=s g(x)=s f(x)=0$ и $x \in \operatorname{Ker}(g)=0$. Поэтому $f-g$-мономорфизм.

(3) Допустим, что существует мономорфизм $f^{\prime}: Y \rightarrow M$. Так как $Y \cong Z$, то существует мономорфизм $g^{\prime}: Z \rightarrow M$. Полагая $f(y+z)=f^{\prime}(z)$ и $g(y+z)=g^{\prime}(z)$, мы продолжим гомоморфизмы $f^{\prime}$ и $g^{\prime}$ до гомоморфизмов $f, g: X \rightarrow M$, где $\operatorname{Ker}(f)=Z$ и $\operatorname{Ker}(g)=Y$. По (2) гомоморфизм $f-g: X \rightarrow M$ является мономорфизмом. Поэтому равномерньй модуль $M$ содержит подмодуль $(f-g)(Y) \oplus(f-g)(Z)$, где $(f-g)(Y)$ и $(f-g)(Z)$ - ненулевые модули. Получено противоречие.

(4) Так как $N$ - вполне инвариантньй подмодуль модуля $M$, то левьй $\operatorname{End}(N)$-модуль $\operatorname{Hom}(X, N)$ является левым $\operatorname{End}(M)$-модулем. Поэтому $\operatorname{Hom}(X, N)$ - подмодуль левого $R$-модуля Безу. Следовательно, $\operatorname{Hom}(X, N)$ - дистрибутивный $R$-модуль.

(5) Так как $M$ - инъективньй модуль и $N$ - квазиинъективньй существенньй подмодуль модуля $M$, то $M$ - инъективная оболочка квазиинъективного модуля $N$. Поэтому $N$ - вполне инвариантный подмодуль модуля $M$ (см. предложение 19.2 из [2]). По (4) $\operatorname{Hom}(X, N)$ - левьй $R$-модуль Безу. Кроме того, каждый эндоморфизм модуля $N$ продолжается до эндоморфизма инъективного модуля $M$. Следовательно, $\operatorname{Hom}(X, N)-$ дистрибутивньй левый $\operatorname{End}(N)$-модуль.

Лемма 2.5. Пусть $X-$ правый модуль над кольцом $A$ и $M-$ такой $X$-инбективный правый $A$-модуль, что левый $\operatorname{End}(M)$-модуль $\operatorname{Hom}(X, M)$ дистрибутивен.

(1) Если $X^{\prime}-$ подмодуль модуля $X$ и существуют такие гомоморфизмы $f^{\prime}, g^{\prime}$ : $X^{\prime} \rightarrow M$, чmo

$$
X^{\prime}=\operatorname{Ker}\left(f^{\prime}\right)+\operatorname{Ker}\left(g^{\prime}\right)
$$

то ядро гомоморфизма

$$
f^{\prime}-g^{\prime}: X^{\prime} \rightarrow M
$$

равно $\operatorname{Ker}\left(f^{\prime}\right) \cap \operatorname{Ker}\left(g^{\prime}\right)$.

(2) Если модуль $M$ равномерен $и X_{1}$ - подмодуль модуля $X$, то для любих двух гомоморфизмов $f_{1}, g_{1}: X_{1} \rightarrow M$ верно, что либо $\operatorname{Ker}\left(f_{1}\right) \subseteq \operatorname{Ker}\left(g_{1}\right)$, либо $\operatorname{Ker}\left(g_{1}\right) \subseteq \operatorname{Ker}\left(f_{1}\right)$. 
ДокАЗАтЕльство. Так как $M-X$-инъективньй правый $A$-модуль, то для любого подмодуля $X^{\prime}$ правого $A$-модуля $X$ существует естественный эпиморфизм дистрибутивного левого $\operatorname{End}(M)$-модуля $\operatorname{Hom}(X, M)$ на левый $\operatorname{End}(M)$-модуль $\operatorname{Hom}\left(X^{\prime}, M\right)$. Поэтому $\operatorname{Hom}\left(X^{\prime}, M\right)$ - дистрибутивньй левый $\operatorname{End}(M)$-модуль. Так как для любого фактор-модуля $\bar{X}$ правого $A$-модуля $X^{\prime}$ существует естественный мономорфизм левого $\operatorname{End}(M)$-модуля $\operatorname{Hom}(\bar{X}, M)$ в дистрибутивньй левьй $\operatorname{End}(M)$-модуль $\operatorname{Hom}\left(X^{\prime}, M\right)$, то $\operatorname{Hom}(\bar{X}, M)$ - дистрибутивньй левьй $\operatorname{End}(M)$-модуль.

(1) Положим

$$
\begin{aligned}
& \bar{X} \equiv X^{\prime} /\left(\operatorname{Ker}\left(f^{\prime}\right) \cap \operatorname{Ker}\left(g^{\prime}\right)\right), \quad \bar{Y}=\operatorname{Ker}\left(g^{\prime}\right) /\left(\operatorname{Ker}\left(f^{\prime}\right) \cap \operatorname{Ker}\left(g^{\prime}\right)\right), \\
& \bar{Z} \equiv \operatorname{Ker}\left(f^{\prime}\right) /\left(\operatorname{Ker}\left(f^{\prime}\right) \cap \operatorname{Ker}\left(g^{\prime}\right)\right) .
\end{aligned}
$$

Так как $X^{\prime}=\operatorname{Ker}\left(f^{\prime}\right)+\operatorname{Ker}\left(g^{\prime}\right)$, то $\bar{X}=\bar{Y} \oplus \bar{Z}$. Гомоморфизмы $f^{\prime}$ и $g^{\prime}$ индуцируют такие гомоморфизмы $\bar{f}, \bar{g}: \bar{X} \rightarrow M$, что

$$
\operatorname{Ker}(\bar{f})=\bar{Z} \quad \text { и } \quad \operatorname{Ker}(\bar{g})=\bar{Y} .
$$

Применим лемму 2.4(2) к модулю $\bar{X}=\bar{Y} \oplus \bar{Z}$ и гомоморфизмам $\bar{f}, \bar{g}: \bar{X} \rightarrow M$. По этой лемме гомоморфизм $\bar{f}-\bar{g}: \bar{X} \rightarrow M$ является мономорфизмом. Поэтому

$$
\operatorname{Ker}\left(f^{\prime}-g^{\prime}\right)=\operatorname{Ker}\left(f^{\prime}\right) \cap \operatorname{Ker}\left(g^{\prime}\right) .
$$

(2) Положим

$$
X^{\prime} \equiv \operatorname{Ker}\left(f_{1}\right)+\operatorname{Ker}\left(g_{1}\right) \subseteq X_{1} .
$$

Обозначим через $f^{\prime}$ и $g^{\prime}$ сужение гомоморфизмов $f_{1}$ и $g^{\prime}$ на модуль $X^{\prime}$. Тогда $\operatorname{Ker}\left(f^{\prime}\right)=$ $\operatorname{Ker}\left(f_{1}\right)$ и $\operatorname{Ker}\left(g^{\prime}\right)=\operatorname{Ker}\left(g_{1}\right)$. По (1) ядро гомоморфизма $f^{\prime}-g^{\prime}: X^{\prime} \rightarrow M$ paвно $\operatorname{Ker}\left(f^{\prime}\right) \cap$ $\operatorname{Ker}\left(g^{\prime}\right)$. Пусть

$$
\begin{aligned}
& \bar{X} \equiv X^{\prime} /\left(\operatorname{Ker}\left(f^{\prime}\right)+\operatorname{Ker}\left(g^{\prime}\right)\right) \bar{Y} \equiv \operatorname{Ker}\left(g^{\prime}\right) /\left(\operatorname{Ker}\left(f^{\prime}\right) \cap \operatorname{Ker}\left(g^{\prime}\right)\right), \\
& \bar{Z} \equiv \operatorname{Ker}\left(f^{\prime}\right) /\left(\operatorname{Ker}\left(f^{\prime}\right) \cap \operatorname{Ker}\left(g^{\prime}\right)\right)
\end{aligned}
$$

Тогда $\bar{X}=\bar{Y} \oplus \bar{Z}$ и гомоморфизм $f^{\prime}-g^{\prime}$ индуцирует мономорфизм $\bar{h}: \bar{Y} \oplus \bar{Z}$ в равномерный модуль $M$. Поэтому либо $\bar{Y}=0$, либо $\bar{Z}=0$. Следовательно, либо $\operatorname{Ker}\left(g^{\prime}\right) \subseteq$ $\operatorname{Ker}\left(f^{\prime}\right)$, либо $\operatorname{Ker}\left(f^{\prime}\right) \subseteq \operatorname{Ker}\left(g^{\prime}\right)$.

Лемма 2.6. Пусть $A$ - кольцо, $X$ - правый $A$-модуль, $S$ - простой правый $A$-модуль и $M$ - инвективная оболочка модуля $S$. Тогда равносильны следующие условия:

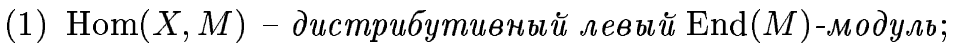

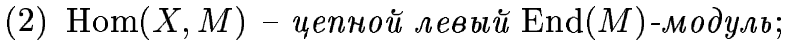

(3) $X$ не имеет подфакторов $S_{1} \oplus S_{2}$ таких, что $S_{1} \cong S_{2} \cong S$.

ДокАЗАТЕЛьСТво. Импликация $(2) \Longrightarrow(1)$ очевидна.

Эквивалентность условий (2) и (3) следует из леммы 1.2.

$(1) \Longrightarrow(2)$ По лемме 1.2 достаточно показать, что для любых двух гомоморфизмов $f$ и $g$ из $X$ в $M$ модули $\operatorname{Ker}(f)$ и $\operatorname{Ker}(g)$ сравнимы по включению. Это следует из леммы $2.5(2)$ и того, что инъективная оболочка простого модуля $S$ является равномерным модулем. 
Лемма 2.7. Пусть $M-$ правый модуль над кольцом $A$. Тогда существует такое множсество $\left\{S_{i}\right\}_{i \in I}$ простых правых $A$-модулей, что модуль $M$ изоморфен подмодулю прямого произведения ингективных оболочек простых модулей $S_{i}$.

Следовательно, каждый инвективный модуль изоморфен прямому слагаемому прямого произведения ингективных оболочек простых модулей.

ДокАЗАТЕЛЬСТво. Пусть $\left\{M_{i}\right\}_{i \in I}$-множество всех ненулевых циклических подмодулей модуля $M$. Для каждого модуля $M_{i}$ сушествует эпиморфизм $f_{i}: M_{i} \rightarrow S_{i}$ на некоторьй простой модуль $S_{i}$. Пусть $E_{i}$ - инъективная оболочка модуля $S_{i}$ и $E=\prod_{i \in I} E_{i}$. Гомоморфизмы $f_{i}$ продолжаются до гомоморфизмов $g_{i}: M \rightarrow E_{i}$, которые индуцируют гомоморфизм $g=\left(g_{i}\right)_{i \in I}: M \rightarrow E$. Так как $g\left(M_{i}\right)=f_{i}\left(M_{i}\right) \neq 0$, то $\operatorname{Ker}(g)=0$.

Лемма 2.8. Для правого модуля $Х$ над кольцом А равносильны следующие условия:

(1) $X-$ - истрибутивный модуль;

(2) для любого простого правого $A$-модуля $S$ с инвективной оболочкой $E$ левый

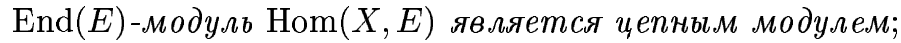

(3) для любого простого правого $A$-модуля $S$ с инвективной оболочкой $E$ левый

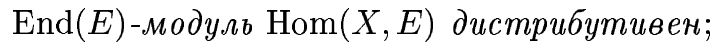

(4) для любого инвективного правого $A$-модуля $M$ левый $\operatorname{End}(M)$-модуль $\operatorname{Hom}(X, M)$ дистрибутивен;

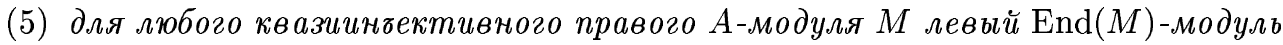
$\operatorname{Hom}(X, M)$ дистрибутивен.

ДоКАЗАТЕЛьСТво. Эквивалентность условий (1) и (2) следует из леммы 1.3.

Эквивалентность условий (2) и (3) следует из леммы 2.6.

$(3) \Longrightarrow(4)$ По лемме 2.7 существует такоемножество $\left\{E_{i}\right\}_{i \in I}$ инъективных оболочек простых правых $A$-модулей, что инъективньй модуль $M$ изоморфен прямому слагаемому модуля $E \equiv \prod_{i \in I} E_{i}$. По условию $\operatorname{Hom}\left(X, E_{i}\right)$ - дистрибутивный левый $\operatorname{End}\left(E_{i}\right)$-модуль для каждого $i \in I$. Так как

$$
\operatorname{Hom}(X, E) \cong \prod_{i \in I} \operatorname{Hom}\left(X, E_{i}\right)
$$

то по лемме 2.3(2) $\operatorname{Hom}(X, E)$ - дистрибутивньй левьй $\operatorname{End}(E)$-модуль. По лемме 2.4(1) $\operatorname{Hom}(X, M)$ - дистрибутивный левьй $\operatorname{End}(M)$-модуль.

Импликации $(4) \Longrightarrow(3)$ и (5) $\Longrightarrow(4)$ очевидны.

Импликация $(4) \Longrightarrow(5)$ следует из леммы $2.4(5)$ и того, что каждый модуль обладает инъективной оболочкой.

Лемма 2.9. Для кольиа А равносильны следующие условия:

(1) $A$ - дистрибутивное справа кольцо;

(2) для любого простого правого $A$-модуля $S$ с инвективной оболочкой $M$ ле-

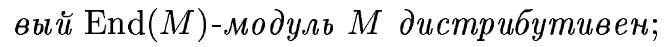

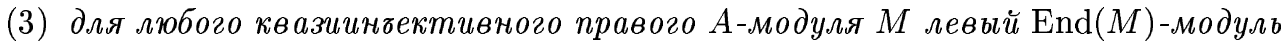
М дистрибутивен. 
ДокАЗАТЕЛЬСТво. Для каждого правого $A$-модуля $M$ существует естественньй изоморфизм $M \cong \operatorname{Hom}\left(A_{A}, M\right)$. Поэтому утверждение следует из леммы 2.8 .

ЛЕмма 2.10. (1) $A$ - дистрибутивное справа полусовершенное кольио тогда и только тогда, когда $A$ - конечное прямое произведение чепных справа колеи.

(2) $A$ - совершенное справа или слева дистрибутивное справа кольцо тогда и только тогда, когда $A$ - конечное прямое произведение цепных справа артиновых справа колеи.

(3) Если $A$ - дистрибутивное справа кольцо и $M-$ правый $A$-модуль, являющийся прямой суммой или прямым произведением квазиинбективных модулей $M_{i}$,

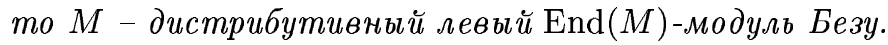

(4) Пусть $A$ - полусовершенное кольцо, каждый неразложимый инвективный правый $A$-модуль $M$ является либо дистрибутивным левым $\operatorname{End}(M)$-модулем, либо левым $\operatorname{End}(M)$-модулем Безу и каждый неразложимый проективный правый $A$-модуль $N$ является либо дистрибутивным левым $\operatorname{End}(N)$-модулем, либо левым $\operatorname{End}(N)$-модулем Безу. Тогда $A$ - конечное прямое произведение иепных колец.

ДокАЗАТЕЛьСТво. Утверждения (1) и (2) доказаны в [5].

(3) По лемме 2.9 и предложению 1.8 каждьй модуль $M_{i}$ является дистрибутивным левым $\operatorname{End}(M)$-модулем Безу. По лемме $2.3 M$ - дистрибутивньй левьй $\operatorname{End}(M)$-модуль Безу.

(4) По лемме 2.9 и предложению $1.8 A$ - дистрибутивное справа кольцо. По (1) $A-$ конечное прямое произведение цепных справа колец $A_{1}, \ldots, A_{n}$. Так как все кольца $A_{i}$ являются неразложимыми проективными правыми $A$-модулями с кольцами эндоморфизмов $A_{i}$, то по условию все локальные кольца $A_{i}$ дистрибутивны слева. По симметричному аналогу утверждения (1) все кольца $A_{i}$ являются цепными слева.

Лемма 2.11. Пусть $A$ - совершенное справа или слева кольцо. Тогда равносильны следующие условия:

(1) каждый правый $A$-модуль $M$ является дистрибутивным левым $\operatorname{End}(M)$ модулем Безу и каждый левый $A$-модуль $N$ является дистрибутивным

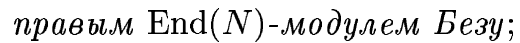

(2) каждый неразложимый инвективный правый $A$-модуль $M$ является либо дистрибутивным левым $\operatorname{End}(M)$-модулем, либо левым $\operatorname{End}(M)$-модулем Безу и каждый неразложимый проективный правый $A$-модуль $N$ является либо дистрибутивным левым $\operatorname{End}(N)$-модулем, либо левым $\operatorname{End}(N)$-модулем Безу;

(3) $A$ - дистрибутивное кольио;

(4) $A$ - конечное прямое произведение иепных артиновых колец.

ДокАЗАТЕЛьСТво. Импликация $(1) \Longrightarrow(2)$ очевидна.

Импликация $(2) \Longrightarrow(3)$ следует из леммы $2.10(4)$.

Импликация $(3) \Longrightarrow(4)$ следует из леммы $2.10(1)$ и леммы $2.10(2)$.

$(4) \Longrightarrow(1)$ Так как $A$ - полуцепное артиново кольцо, то каждьй $A$-модуль является прямой суммой квазиинъективных модулей (см., например, [4, п. 55.16]). Кроме того, $A$ - дистрибутивное кольцо. Поэтому утверждение следует из леммы 2.10(3). 
ОКОНЧАНИЕ ДОКАЗАТЕЛЬСТВА тЕОРЕмы 2. Теорема 2 следует из леммы 2.11.

ПРИмеР 2.12. Пусть $\mathbb{Z}$ - кольцо цельх чисел. Тогда $\mathbb{Z}$ - дистрибутивное кольцо, над которым не все модули являются дистрибутивными модулями над своими кольцами эндоморфизмов.

ДокАЗАТЕльство. Дистрибутивность кольца $\mathbb{Z}$ хорошо известна и проверяется непосредственно. По теореме 88.4 из [6] существует такая абелева группа $M$, что $M-$ неразложимая группа без кручения ранга 2 и все эндоморфизмы группы $M$ являются умножениями на целые числа. Тогда множество всеx $\operatorname{End}(M)$-подмодулей группы $M$ совпадает со множеством всех ее $\mathbb{Z}$-подмодулей. Группа $M$ не является дистрибутивным $\mathbb{Z}$-модулем, поскольку $M$ содержит прямую сумму двух изоморфных ненулевых подгрупш. Поэтому $M$ не является дистрибутивным $\operatorname{End}(M)$-модулем.

\section{СПИСОК ЦИТИРОВАННОЙ ЛИТЕРАТУРЫ}

[1] Tuganbaev A. A. Semidistributive Modules and Rings. Dordrecht-Boston-London: Kluwer Academic Publishers, 1998.

[2] Фейс К. Алгебра: кольца, модули и категории. II. М.: Мир, 1979.

[3] Camillo V. Distributive modules // J. Algebra. 1975. V. 36. № 1. P. 16-25.

[4] Wisbauer R. Foundations of Module and Ring Theory. Philadelphia: Gordon and Breach Science Publishers, 1991.

[5] Stephenson W. Modules whose lattice of submodules is distributive // Proc. London Math. Soc. 1974. V. 28. № 2. P. 291-310.

[6] Фукс Л. Бесконечные абелевы группы. Т. II. М.: Мир, 1977.

Московский энергетический институт (технический университет) 